\title{
2 Erkennen eines potentiellen Spenders
}

\author{
Klaus Hahnenkamp \\ Die Erkennung potentieller Organspender ist der Schlüsselpro- \\ zess in der postmortalen Organspende.
}

Potentielle Organspender sind beatmete Patienten mit primärer oder sekundärer akuter schwerer Hirnschädigung, die nach ärztlicher Beurteilung als Organspender in Betracht kommen. Gemäß $₫$ ga Abs. 2 Nr. 1 TPG sind Entnahmekrankenhäuser verpflichtet, den irreversiblen Hirnfunktionsausfall festzustellen. Der Terminus ärztliche Beurteilung richtet sich maßgeblich nach medizinischen Kriterien, aber nicht ausschließlich. So ist ein bereits prämortal offenbarter Widerspruch erheblich. Im letzten Fall ist ein Patient kein (potentieller) Organspender und die postmortale Organspende ausgeschlossen. Ansonsten muss ein möglicher Organspendewille eines Patienten im Behandlungsablauf mit bedacht werden.

„Patientenvertreter sind im Behandlungsverlauf darüber zu informieren, wenn eine Fortführung der intensivmedizinischen Maßnahmen nicht mehr primär zum Wohle des Patienten, sondern zur Realisierung einer möglichen Organspende erwogen wird. Es sollte über die klinische Einschätzung und über mögliche weitere Schritte gesprochen werden um dem Willen des Patienten zu entsprechen. Das Gespräch sollte bereitsstattfinden, wenn der Eintritt des irreversiblen Hirnfunktionsausfalls wahrscheinlich ist." (Zentrale Ethikkommission bei der Bundesärztekammer 2013) 
Dies geschieht primär unabhängig von der Organspende und dient der Festlegung von Therapiezielen und intensivmedizinischen Maßnahmen. Die rechtswirksame Einwilligung zur Organspende ist nach dem Transplantationsgesetz (TPG) immer postmortal festzustellen bzw. abschließend postmortal zu bestätigen.

Kommt ein potentieller Organspender zugleich als Gewebespender in Betracht, so soll die Gewebespende gemäß Transplantationsgesetz zusammen Beachtung finden ( $\$ 4 \mathrm{Abs}$. $1 \mathrm{TPG}$ ). Die mögliche postmortale Entnahme und Übertragung eines Organs hat allerdings Vorrang vor der postmortalen Entnahme von Geweben (Subsidiaritätsprinzip), insbesondere darf eine Organspende nicht durch eine Gewebespende beeinträchtigt werden ( $\$ 9$ Abs. 3 TPG).

Potentielle Organspender sind beatmete Patienten, die nach ärztlicher Beurteilung als postmortale Organspender in Betracht kommen. Zur primären ärztlichen Beurteilung müssen folgende medizinische und nicht medizinische Voraussetzungen erfüllt sein:

- schwere akute primäre oder sekundäre Hirnschädigung vorliegend

- prima vista kein Widerspruch gegen die Organspende erkenntlich

- bis dato keine absoluten medizinischen Kontraindikationen vorbekannt

- irreversibler Hirnfunktionsausfall festgestellt, vermutet, eingetreten oder unmittelbar bevorstehend

\subsection{Voraussetzung des Erkennens eines potentiellen Spenders} Gerold Söffker

\subsubsection{Terminus potentieller Organspender}

Der Terminus „potentieller Organspender“ ist im Transplantationsgesetzt, vielleicht aus gutem Grund, nicht ausdrücklich erwähnt, ergibt sich aber indirekt aus den dortigen Vorgaben. Der Terminus „möglicher Organspender“ wird bereits für die rein elektronisch („Transplantcheck“) aus den Abrechnungsdaten retrospektiv gefilterten Patienten verwendet (Schulte et al. 2018).

In der Literatur werden zur prospektiven Identifikation von Organspendern weitere Begrifflichkeiten im Organspendeprozess vorgeschlagen (Dominguez-Gil et al. 2011; Guide to the Quality and Safety of Organs for Transplantation 2018). Unabhängig von der Begrifflichkeit muss primär ein Patient mit „verheerender“ Hirnverletzung, unmittelbar bevorstehendem vollständigen 
Hirnfunktionsausfall und mutmaßlicher medizinischer Eignung im ersten Prozessschritt vom ärztlichen Behandlungsteam detektiert werden. In der internationalen Literatur werden dabei keine einheitlichen medizinischen Kriterien und klinische Trigger zur Erkennung eines potentiellen postmortalen Organspenders („possible organ donor“) genannt (Squires et al. 2018). Im nächsten Prozessschritt muss der vollständige Hirnfunktionsausfall als eingetreten vermutet werden („potential donor“) und anschließend der irreversible Hirnfunktionsausfall gesetzeskonform festgestellt werden („eligible donor“). Diese Teilschritte im Organspendeprozess werden in der Richtlinie unter dem Überbegriff des potentiellen Organspenders und dessen Management abgebildet.

\subsubsection{Zu welchem Zeitpunkt und wo werden potentielle postmortale Organspender identifiziert?}

Das Transplantationsgesetzt legt weder den Ort noch den frühesten Zeitpunkt ausdrücklich fest. Die Erkennung von potentiellen Organspendern erfolgt prinzipiell ab Krankenhausaufnahme, z.B. bereits im Schockraum, zumeist aber erst auf der Intensivstation und nach Verlaufsbeobachtung. Sobald Patienten mit akuter schwerer Hirnschädigung bekannter Ätiologie und ohne medizinische Kontraindikationen für eine Organspende Alarmzeichen für einen Hirnfunktionsausfall entwickeln, müssen diese als potentielle Organspender identifiziert werden. Die frühzeitige Identifikation von potentiellen Organspendern ist notwendig (Martin-Loeches et al. 2019), allerspätestens vor einer Therapielimitierung muss diese Identifikation erfolgt sein.

Die „Ethicus-2 Studie“ (Sprung et al. 2019) konnte aufzeigen, dass in der Studienkohorte bei 89,7\% der erwachsenen intensivpflichtigen Patienten von 22 europäischen Intensivstationen eine Therapievorenthaltung, Therapiebeendigung oder aktive Verkürzung des Sterbeprozesses (primärer Endpunkt) zu beobachten war. Die mediane Zeit von intensivmedizinischer Aufnahme bis zur ersten Limitierung einer lebenserhaltenden Therapie betrug hierbei 2,1 Tage. Diese Daten zeigen, dass es insgesamt zu einem Wandel in der Therapie am Lebensende, unter Einschluss von Therapiebegrenzungen, sowie zu einem Rückgang in der Feststellung des irreversiblen Hirnfunktionsausfalls gekommen ist (Janssens 2020). Im Kontext der Organspende muss daher bei Entscheidungen am Lebensende („end-of-life decisions“) und insbesondere vor Therapielimitierung auch ein möglicher Organspendewunsch bei potentiellen Organspendern eruiert werden, ansonsten kann diesem Wunsch keine Geltung mehr verschafft werden.

Erfolgen in Ausnahmefällen entgegen der Vorgaben der Richtlinie Gespräche zu Therapie- und Therapieziel-Entscheidungen am Lebensende nicht oder 
können in besonderen klinischen Situationen aus ärztlicher Sicht nicht erfolgen, so muss spätestens nach Feststellung des irreversiblen Hirnfunktionsausfalls bei allen potentiellen Organspendern die Evaluation des Organspendewunsches erfolgen.

\subsubsection{Verhältnis Patientenverfügung und Organspende}

Mit dem „Gesetz zur Verbesserung der Rechte von Patientinnen und Patienten" hat die Legislative 2013 die Patientenrechte gestärkt. Demnach bedarf es z.B. bei fehlenden therapeutischen Therapieoptionen („infauste Prognose") bei vorliegender Patientenverfügungen oder anderen Willensbekundungen zur Therapiebegrenzung nicht erst einer Todesfeststellung, um aus Patientensicht „sinnlose“ Therapiemaßnahmen zu beenden. Allerdings beantwortet das alleinige Vorliegen z.B. einer Patientenverfügung zumeist nicht auch die Frage nach einem Organspendewunsch (Zentrale Ethikkommission bei der Bundesärztekammer 2013). Um hierbei eine vom Patienten auch gewollte Organspende ermöglichen zu können, muss die Organspenderidentifikation und Organspendeoption in Therapie- und TherapiezielEntscheidungen am Lebensende einbezogen werden (Hahnenkamp et al. 2016). Dieses ist ein Paradigmenwechsel.

Sobald Patienten mit schwerer akuter Hirnschädigung bekannter Ätiologie und ohne vorbekannte medizinische Kontraindikationen „Alarmzeichen“ für einen Hirnfunktionsausfall entwickeln, müssen diese als potentielle Organspender identifiziert werden. Bei potentiellen Organspendern soll prämortal zum klinischen Zeitpunkt von Therapie- und Therapieziel-Entscheidungen am Lebensende und vor Therapielimitierung ein möglicher Organspendewunsch ermittelt und die Organspendeoption frühzeitig mit einbezogen werden; spätestens jedoch nach Feststellung des irreversiblen Hirnfunktionsausfalls.

\subsubsection{Intubation und elektive Beatmung von Patienten ohne therapeutische Therapieoption?}

Potentielle Organspender im Sinne der Richtlinie sind beatmete Patienten, daher kann die Richtlinie dazu keine Regelungen treffen. Von der Richtlinie und aus dem TPG lässt sich die Frage daher nicht beantworten, ob primär nicht intubierte Patienten (insbesondere in der Notaufnahme) mit schwerster akuter Hirnschädigung und ohne therapeutische Option, aber erwartbaren zeitnahen Progress in ein finales Stadium, allein zur Aufrechterhaltung einer Organspendemöglichkeit oder deren Klärung elektiv intubiert 
werden dürfen. Eine nicht therapeutisch intendierte Intubation mit anschließender mechanischer Beatmung und Anwendung intensivmedizinischer Maßnahmen ist in dieser Situation umstritten - sie dient nicht dem Wohle des Patienten, könnte aber dennoch seinem Willen entsprechen. Die Frage nach dem Vorgehen kann daher klinisch relevant sein. Medizinisch im Vordergrund steht primär die Sicherheit der medizinisch infausten Prognoseabschätzung zum Entscheidungszeitpunkt. Insbesondere in der Schockraum-/Notaufnahmesituation kann eine infauste Prognoseabschätzung bei nicht beatmeten Patienten jedoch unsicher sein.

In der internationalen Literatur wird gemäß Kapitel 2.4 des „Guide to the Quality and Safety of Organs for Transplantation“ (2018) eine nicht therapeutisch intendierte Intubation mit einer zeitlich sehr befristeten Klärung der Organspende in dieser speziellen Situation nicht ausgeschlossen. Die Richtlinie und das TPG schliesst ein solches Vorgehen ebenfalls nicht aus. Auf der anderen Seite bedarf dieses Vorgehen, insbesondere aus rechtlicher und ethischer Sicht der Einwilligung (Escudero et al. 2017; Gardiner et al. 2019). Auch ein entgegenstehender Wille zur Therapiebegrenzung oder die Eingriffsintensität der geplanten erweiterten Behandlungsmaßnahmen ist von Belang. Ein sofortiges Gespräch mit den Patientenvertretern wird dringend empfohlen.

\subsubsection{Was sind absolute medizinische Kontraindikationen bei potentiellen Organspendern?}

Medizinische Kontraindikationen schließen eine Organspende aus. So liegt bei Patienten mit chronischen Organversagen für das Einzelorgan sowie bei therapierefraktären Patienten mit progredient verlaufendem schwerem Multiorganversagen oder schwerem Kreislaufschock eine Kontraindikation vor. Die Frage nach weiteren medizinischen Kontraindikationen für eine Organspende ist nur im Kontext der medizinischen Situation des Transplantatempfängers zu beantworten. Was für den einen Empfänger ein unverhältnismäßig hohes Transplantationsrisiko darstellt, kann für einen anderen Empfänger auch bei erhöhtem Risiko die letzte lebensrettende Chance sein. Insbesondere bei akuten oder früheren Tumorerkrankungen des Spenders (Therapie, Histologie, Staging z.B. anhand TNM-Klassifikation und Grading sind wichtig zur Risikoabschätzung) oder Hepatitis C kann es schwer zu bestimmen sein, wo die absoluten Risiken und Grenzen liegen. Die medizinische Nutzen-Risiko-Analyse für den Empfänger ist abschließend nur als Einzelfallentscheidung in der Gesamtschau aller Befunde im Transplantationszentrum organspezifisch zu bestimmen. Zur Risikoabschätzung dienen insbesondere die Anamnese, die körperliche Untersuchung sowie insbesondere die abschließenden postmortalen Untersuchun- 
gen eines Organspenders gemäß der Richtlinie „Empfängerschutz und TPGOrganV“. Diese Untersuchungen bedeuten aber, zusammen mit der allgemeinen Spenderevaluation, auch aus Sicht des Entnahmekrankenhauses einen hohen personellen, zeitlichen und kostenintensiven Ressourceneinsatz. Zudem ist die Gesamtsituation mindestens für die Angehörigen, aber auch für das behandelnde Klinikteam emotional belastend. Erscheint eine abschließend realisierbare Organspende nach medizinischen Kriterien ausgeschlossen bzw. hochwahrscheinlich nicht realisierbar, so stellt sich im Entnahmekrankenhaus die Frage nach der medizinischen Sinnhaftigkeit und Verhältnismäßigkeit. Gerade im Vorfelde einer noch allgemeinen Evaluation eines Patienten mit vermuteten erweiterten Spenderkriterien (,Risiko-Spender“) empfiehlt sich daher frühzeitig die Rücksprache mit dem Transplantationsbeauftragten und eine allgemeine Beratung durch die Koordinierungsstelle (Deutsche Stiftung Organtransplantation). Vorbefunde, oder aus anderen Gründen erlangte aktuelle diagnostische Befunde, dürfen selbstverständlich zur allgemeinen Evaluation auch vor einer Todesfeststellung in Grundsatzüberlegungen zur allgemeinen medizinischen Spendertauglichkeit einbezogen werden. Der „Guide to the Quality and Safety of Organs for Transplantation“ (2018) erlaubt zudem eine Übersicht zur Einschätzung von Kontraindikationen.

Altersunabhängig können z.B. gemäß dem „Guide to the Quality and Safety of Organs for Transplantation“ (2018) folgende allgemeine absoluten Kontraindikationen angegeben werden:

- Aktive oder nicht kurativ behandelte maligne Neoplasie (Ausnahmen sind Primärtumore des zentralen Nervensystems, die keine Metastasen bilden) mit Ausbreitung auf mehrere Organe.

- Unbehandelte oder unkontrollierte schwere systemische Infektionen (z.B. Sepsis mit 4 MRGN Pseudomonas oder Acinetobacter baumannii, HIV, aktive Tuberkulose, aktive SARS-CoV-2 Infektion), insbesondere bei unbekanntem Erreger (z.B. auch unklare Enzephalitis) ohne Therapieansprechen sowie ohne Behandlungsoption beim Empfänger (z.B. Prionen-Erkrankungen, Rabiesvirus).

Kontraindikationen können generell oder nur organspezifisch bestehen. Absolute allgemeine medizinische Kontraindikationen des Spenders liegen vor bei:

- akut progredient verlaufendem schwerem Multiorganversagen oder therapierefraktärem schwerem Kreislaufschock jeglicher Genese

- aktiver, metastasierter oder nicht kurativ behandelter maligner Neoplasie (Ausnahmen möglich) 
- unbehandelter oder unkontrollierter schwerer systemischer Infektion, insbesondere bei unbekanntem Erreger ohne Therapieansprechen sowie ohne Behandlungsoption beim Empfänger

\section{Dringende Empfehlung: Kontraindikationen mit der Koordinie-} rungsstelle besprechen.

\subsubsection{Was meint vollständiger Hirnfunktionsausfall bereits vermutet, eingetreten oder unmittelbar bevorstehend?}

Der vollständige und irreversible Hirnfunktionsausfall wird gemäß der hierzu eigenen Richtlinie festgestellt. Zur Beschreibung der klinischen Situationen, die zur abschließenden Feststellung des irreversiblen Hirnfunktionsausfalls beim potentiellen Organspender führen kann, wurde die begriffliche Unterscheidung zwischen unmittelbar bevorstehendem und vermuteten irreversiblen Hirnfunktionsausfall in die Richtlinie Spendererkennung übernommen. Diese sind bereits in der Gesetzesbegründung zum 2. TPG Änderungsgesetz sowie im Arbeitspapier der Bundesärztekammer zum Verhältnis von Patientenverfügung und Organspendeerklärung angelegt (Zentrale Ethikkommission bei der Bundesärztekammer 2013).

\section{Vermuteter Hirnfunktionsausfall}

Der vollständige Hirnfunktionsausfall wird vermutet, wenn klinische Zeichen einer vollständig ausgefallenen Hirnfunktion mutmaßlich vorliegen. Beim potentiellen Organspender ist konsekutiv die Einleitung der Diagnostik zur endgültigen Feststellung des irreversiblen Hirnfunktionsausfalls obligat.

\section{Unmittelbar bevorstehender Hirnfunktionsausfall}

Der Hirnfunktionsausfall besteht unmittelbar bevor, wenn noch nicht alle klinischen Zeichen eines vollständiges Hirnfunktionsausfalls vorliegen, dieser aber hochwahrscheinlich eintreten wird („,drohender Tod“). Dieses kann prognostiziert werden, wenn bei beatmeten Patienten mit akuter schwerer Hirnschädigung bekannter Genese in der Verlaufsbeobachtung die Ausprägung und Anzahl pathologischer klinischer Befunde, zumeist unterstützt durch zerebrale Bildgebung und weitere apparative diagnostische Methoden (SEP, EEG, Doppler- und Duplexsonographie u.a.) stetig zunehmen. Wie viele klinische Befunde anfänglich mindestens in die gleiche Richtung weisen sollten, ist in der Literatur uneinheitlich und variiert u.a. zwischen 
1-3 neurologischen Ausfällen aus 5-7 klinisch prüfbaren Hirnstammreflexen. Insgesamt werden gemäß Literatur unterschiedliche klinische Kriterien herangezogen (Squires et al. 2018).

Prognostische Aussagen erfolgen anhand medizinischer Befunde und Verläufe, statistischer Wahrscheinlichkeiten, allgemeiner und persönlicher ärztlicher Erfahrung sowie immer mit der gebotenen Sorgfalt. Zu obigem Zeitpunkt besteht, je nach klinischen Befunden, ggf. noch Unsicherheit in der Prognoseeinschätzung.

\section{Der Hirnfunktionsausfall}

- wird gemäß Richtlinie festgestellt

- wird vermutet, wenn klinische Zeichen einer vollständig ausgefallenen Hirnfunktion vorliegen, die Feststellung aber (noch) nicht erfolgt ist

- steht unmittelbar bevor, wenn die klinischen Zeichen (noch) nicht vollständig vorliegen, aber die Ausprägung und Anzahl pathologischer klinischer Befunde stetig zunehmen und konsekutiv der zeitnahe Eintritt hochwahrscheinlich ist

Im anglo-amerikanischen wird für einen unmittelbar bevorstehenden Hirnfunktionsausfall (z.B. vom „Organ Procurement and Transplantation Network“ - OPTN) der Begriff ,imminent neurological death“ verwendet. Allerdings setzt diese Definition voraus, dass z.B. keine Sedation oder Hypothermie die klinische neurologische Einschätzung verhindern (OPTN 2017). Durch die Richtlinie Spendererkennung soll allerdings ermöglicht werden, dass die erste Spenderidentifikation, die Verlaufsbeobachtung und erste klinische Einschätzung des potentiellen Organspenders bereits unter therapeutischen Therapiemaßnahmen beginnen kann. Die klinische Beurteilung und Prognoseabschätzung kann dabei zunächst noch eingeschränkt sein sowie die weitere Therapie und Verlaufsbeobachtung indiziert sein. Die zusätzliche Anwendung von klinischen Scores (z.B. „Glasgow Coma Score“- GCS; „World Federation of Neurosurgical Societies grading system“ - WFNS-Scale; „Full Outline of UnResponsiveness Score“ - FOUR-Score) hat ebenso die Einschränkung der sicheren Aussagekraft unter Sedierung oder Hypothermie. Dennoch können Scores in der klinischen Verlaufsbeobachtung zusätzlich hinweisend sein. Dieses gilt auch für andere vorgeschlagene allgemeine klinische Trigger bei Patienten mit schwerer Hirnschädigung, wie z.B. dem Akronym „GIVE“ (GCS $\leq 5$, intubiert, ventiliert, Entscheidung am Lebensende) (Zavalkoff et al. 2019).

Steht der vollständige Hirnfunktionsausfall unmittelbar bevor, sollte weiterhin die therapeutische Behandlung zum Wohle des Patienten und gemäß 
dem Willen des Patienten durchgeführt werden. In dieser Situation erfolgen dennoch zeitgleich

1. die Behandlung,

2. Verlaufsbeobachtung und

3. weitere potentielle Spenderbeurteilung sowie

4. erste Prognosegespräche und Therapiezielgespräche mit den Patientenvertretern.

Für diese Gespräche sind primär Willensbekundungen des Patienten zu therapeutischen Maßnahmen, zu Entscheidungen am Lebensende und auch zur Organspende relevant. Schriftliche Willensbekundungen zur Organspende können vom Patienten in einer Patientenverfügung, einem (zusätzlichen) Organspendeausweis oder ab März 2022 (zusätzlich) im bundesweiten Online-Organspenderegister erfolgt sein. Darüber hinaus können die Entscheidungen einer dritten Person übertragen worden sein, mündlich mitgeteilt oder überhaupt nicht erfolgt sein. Die Einsichtnahme in das OnlineOrganspenderegister darf gemäß dem „Gesetz zur Stärkung der Entscheidungsbereitschaft bei der Organspende“ (2020) nur durch einen als auskunftsberechtigt benannten Arzt oder Transplantationsbeauftragten erfolgen. Die Registerinformation ist nicht erst nach Feststellung des Todes einsehbar, wie ursprünglich geplant, sondern wird nun so geregelt, dass eine Auskunft bereits in Behandlungssituationen, in denen der nicht behebbare Ausfall der Gesamtfunktion des Großhirns, des Kleinhirns und des Hirnstamms des möglichen Organ- oder Gewebespenders unmittelbar bevorsteht oder als bereits eingetreten vermutet wird, erfragt werden darf, um diese Information an die behandelnden Ärzte für Therapiezielgespräche am Lebensende weiterzugeben.

Komplex ist die Festlegung eines Therapieziels insbesondere dann, wenn aufgrund einer rechtswirksamen Patientenverfügung eine Behandlungsbegrenzung gewünscht wird, zudem aber auch ein möglicher Organspendewunsch besteht. Dieser muss im Rahmen der Entscheidungen am Lebensende (,end-of-life decisions“, partizipative Entscheidungsfindung) sodann erkundet werden, sonst könnte der ebenso bestehende Organspendewunsch niemals umgesetzt werden. Bei divergent erscheinenden Willensbekundungen (z.B. palliative care vs. Organspendewunsch) erfolgt die harmonisierende Interpretation aller Willensäußerungen durch den Patientenvertreter (Betreuer, Vorsorgebevollmächtigten) sowie danach die Priorisierung des höchsten Willens für diese Gesamtsituation. Ist der Wille zur Therapiebegrenzung ausdrücklich oder priorisiert, muss dieser umgesetzt werden. Wird der Wille zur Organspende priorisiert, muss diesem Wunsch gefolgt werden. In der Regel wird hierfür ein zeitlicher Rahmen mit den Patientenvertretern besprochen. 
Sind postmortal mehrere und sich widersprechende Erklärungen pro und kontra Organspende bekannt, gilt die zuletzt abgegebene Erklärung. Ist postmortal nicht festzustellen und subsidiär dem nächsten Angehörigen nicht bekannt, welche Erklärung zuletzt abgegeben worden ist, gilt diejenige Erklärung mit der geringsten Eingriffstiefe ( $\$ 2 \mathrm{Abs} .2 \mathrm{TPG})$. Letzteres heißt im Klartext: im Zweifelsfall keine postmortale Organspende.

Schriftliche Willensbekundungen zum prämortalen Umfang einer Behandlung und zur postmortalen Organspende können aus einer Patientenverfügung, dem Organspendeausweis oder aus dem Eintrag im online Organspenderegister ersichtlich sein.

- Prämortal erfolgt durch den Patientenvertreter (Betreuer, Vorsorgebevollmächtigten) die harmonisierende Interpretation aller eruierbaren ausdrücklichen (schriftlich, mündlich) oder mutmaßlichen Willensäußerungen des Patienten sowie bei divergierenden Willensbekundungen die Priorisierung eines führenden Gesamtwillens.

- Bei postmortal mehreren, sich widersprechenden Organspendeerklärungen gilt die zuletzt abgegebene Erklärung. Ist postmortal auch durch Befragung der nächsten Angehörigen nicht festzustellen, welche Erklärung zuletzt abgegeben worden ist, gilt diejenige Erklärung mit der geringsten Eingriffstiefe, d.h. im Zweifelsfall keine postmortale Organspende.

\subsubsection{Wie lang kann beim unmittelbar bevorstehenden vollständigen Hirnfunktionsausfall auf dessen Eintritt gewartet werden?}

Steht der Hirnfunktionsausfall unmittelbar bevor und besteht prinzipiell ein Organspendewunsch des Patienten, so kann in obigen Diskussionen die Zeitspanne der weiteren Verlaufsbeobachtung bis hin zur Feststellung des irreversiblen Hirnfunktionsausfalls eine wesentliche Bedeutung für Entscheidungen am Lebensende erlangen. Für diese Zeitspanne besteht keine gesetzliche Vorgabe im Transplantationsgesetz.

Die Zeitspanne richtet sich immer zuerst nach dem Patientenwillen. Darüber hinaus erscheinen medizinische (z.B. Kontraindikationen, Herz-Kreislaufstabilität, Ausprägung des erweiterten intensivmedizinischen Behandlungsbedarfs gemäß Neitzke et al. 2019), ethische (z.B. Menschenwürde sowie die vier Prinzipien nach Childress und Beauchamp 2008): Patientenautonomie, Benefizienz, Nonmalefizienz und Gerechtigkeit), verfahrenstechnische und vergleichende Überlegungen sinnvoll. Darüber hinaus ist die Prognosesicherheit zu beachten. In anderen klinischen Situationen, die 
aber auch für die Organspende relevant sein können, empfiehlt z.B. die S1Leitlinie „Hypoxisch-ischämische Enzephalopathie“ bei reanimierten $\mathrm{Pa}$ tienten mit Temperaturmanagement die neurologische Prognoseabschätzung nach $72 \mathrm{~h}$ zu beginnen (Bender et al. 2018). In Situationen mit schwerer neurologischer Schädigung wird allgemein kongruent eine Verlaufsbeobachtung über 48-72 h empfohlen (Souter et al. 2015; Harvey et al. 2018; Rohaut u. Claassen 2018). Im Hinblick auf die Organspende ist rein verfahrenstechnisch gemäß der Richtlinie zur Feststellung des irreversiblen Hirnfunktionsausfalls (vierte Fortschreibung) ab Beginn des dritten Lebensjahres und bei vorliegender sekundärer Hirnschädigung zum Irreversibilitätsnachweis anhand klinischer Symptome eine Zeitspanne $\geq 72 \mathrm{~h}$ erforderlich. Ebenso vergleichend wurde im zuletzt 2017 revidierten Transplantationsgesetz der Schweiz bzw. den dazugehörigen medizin-ethischen Richtlinien der Schweizerischen Akademie für Medizinische Wissenschaften (SAMW), gegenüber 2011, von einer zeitlichen Limitierung für medizinische Maßnahmen vor dem Tod ganz abgesehen. In Hinweisen zur praktischen Umsetzung wird allerdings eine Zeitspanne von $48 \mathrm{~h}$ (danach Donation after Circulatory Death) genannt (SAMW 2017). Im „Guide to the Quality and Safety of Organs for Transplantation“ (2018, S. 53). wird eine Beobachtungszeit von $72 \mathrm{~h}$ angeführt.

Zusammenfassend erscheint eine medizinische Verlaufsbeobachtung, sofern dieses dem Patientenwillen nicht widerspricht, bis ca. $72 \mathrm{~h}$ bei Patienten mit schwerer Hirnschädigung konsensfähig. In der Richtlinie erfolgte keine zeitliche Präzisierung.

Bei potentiellen Organspendern und unmittelbar bevorstehendem vollständigen Hirnfunktionsausfall erscheint, sofern dieses dem Patientenwillen nicht widerspricht, eine Beobachtungszeit von ca. 48-72 $h$ konsensfähig.

\subsubsection{Welche Struktur benötigt ein Entnahmekrankenhaus zur potentiellen Spendererkennung?}

Die übergeordnete Struktur der Organspende und Transplantationsmedizin ist im Transplantationsgesetz (TPG) festgelegt. Die Erkennung potentieller Organspender im Entnahmekrankenhaus ist keine Einzelleistung und nur erfolgreich umsetzbar, wenn von Seiten der Krankenhausleitung eine innerklinische Kultur der Organspende aktiv unterstützt wird. Die dazu notwendigen internen Strukturen sind Grundlage zur potentiellen Spendererkennung (Leitpfad zur potentiellen Spendererkennung und Sequenz in der Organspende siehe Abbildung 1 und 2). 


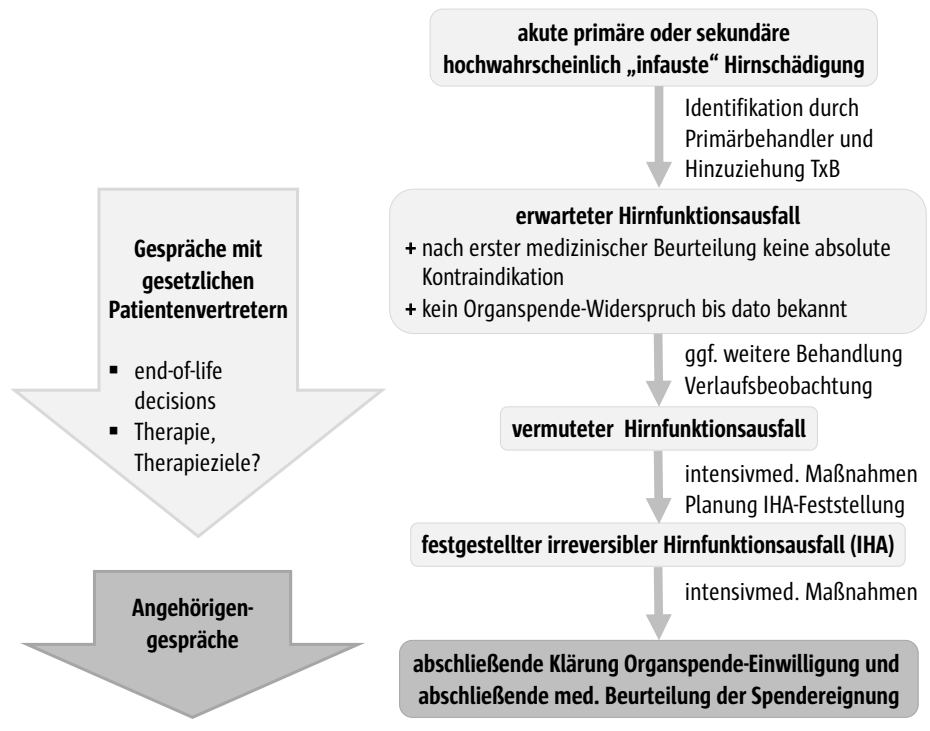

Abb. 1 Leitpfad zur Erkennung von potentiellen Organspendern

Das Entnahmekrankenhaus ist verpflichtet, den irreversiblen Hirnfunktionsausfall bei allen potentiellen Organspendern festzustellen und diesen immer unverzüglich der Koordinierungsstelle (DSO) mitzuteilen. Zur Sicherstellung dieser Verpflichtung sind krankenhausinterne Verfahrensanweisungen, welche Zuständigkeiten und Handlungsabläufe regeln, vorgeschrieben (\$ 9a Abs. 2 TPG). Zur Organspende informiertes und qualifiziertes Krankenhauspersonal nimmt die Aufgaben im Organspendeprozess wahr. Hierbei hat der unabhängige und qualifizierte Transplantationsbeauftragte eine besondere Stellung. Dieser muss immer hinzugezogen werden, wenn Patienten nach ärztlicher Beurteilung als Organspender in Betracht kommen ( $\$$ gb Abs. 1 TPG). Der Transplantationsbeauftragte hat zur Wahrung seiner Aufgaben Zugangsrecht zur Intensivstation oder Notaufnahme. Die primäre Erkennung potentieller Organspender ist der Schlüsselprozess zur Spenderidentifikation und es liegt in der Primärverantwortung aller Ärzte und Pflegenden, die diese Patienten behandeln (Primärbehandlungsteam), solche Patienten zu erkennen und den Transplantationsbeauftragten hinzuzuziehen. Transplantationsbeauftragte werden in keinem Entnahmekrankenhaus rund um die Uhr unverzüglich verfügbar sein können. Die Expertise zur ersten potentiellen Spendererkennung sollte auf jeder einzelnen Intensivstation sowie übergeordnet in der gesamten Intensiv- und Notfallmedizin, in Schlaganfall- oder Traumazentren sowie in Cardiac-Arrest-Zentren vorhanden sein. 


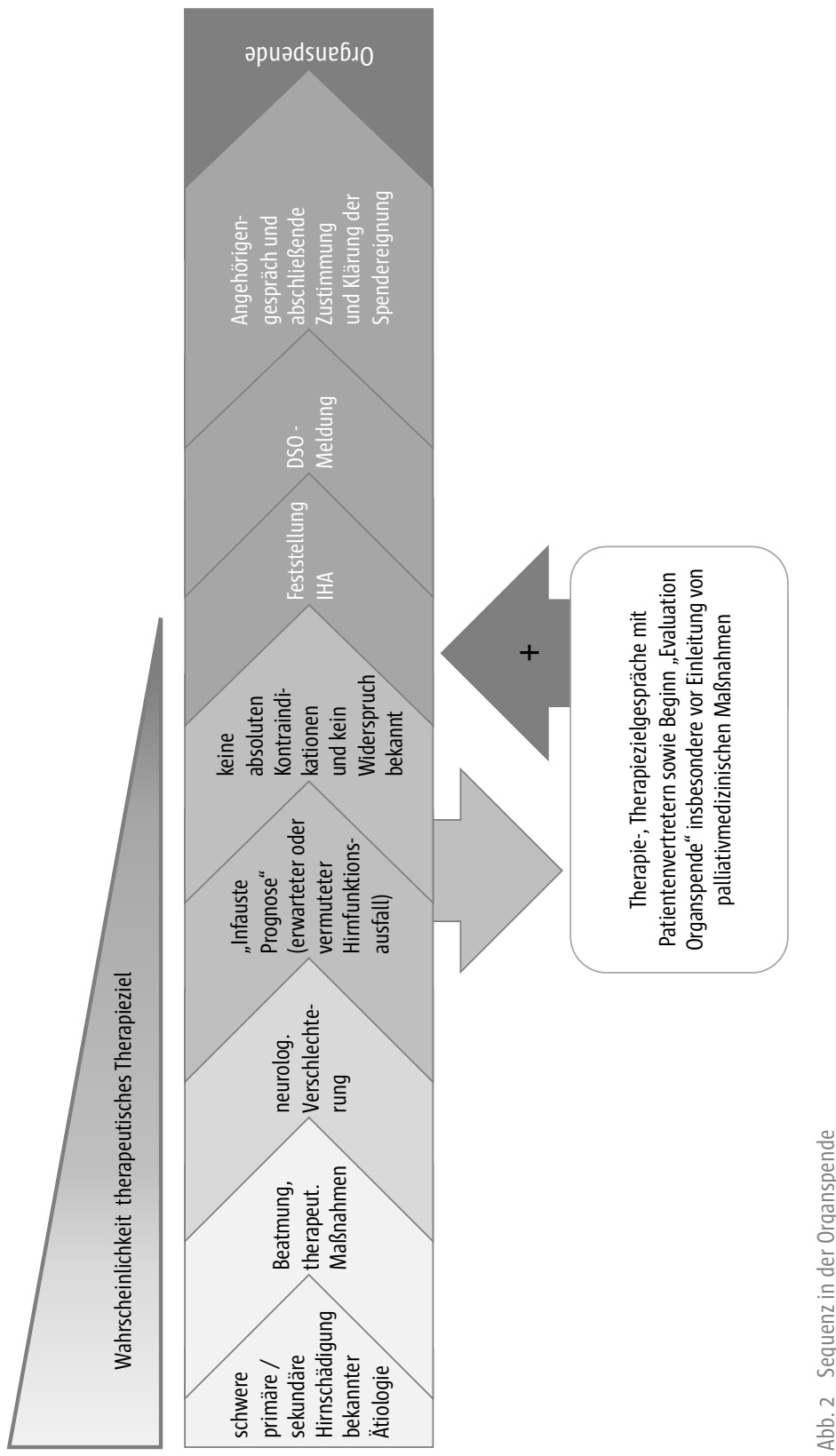


Insbesondere Alarmzeichen (,brain death alert signs“) sollten von jedem Intensivmediziner erkannt werden können (Martin-Loeches et al. 2019). Checklisten können hierbei in der Erkennung und im Management hilfreich sein.

Zur Prozesskontrolle (Qualitätssicherung) müssen für jeden Einzelfall alle Todesfälle mit kodierter primärer oder sekundärer Hirnschädigung im Krankenhaus retrospektiv softwarebasiert analysiert („Transplantcheck“), dokumentiert sowie intern wie extern berichtet werden. Darüber hinaus könnte sich auch eine in die tägliche Routine implementierte Analyse, z.B. im Rahmen von Morgen- oder Mittagsbesprechungen sowie Morbiditäts- und Mortalitätskonferenzen, anbieten. Zur Realisierung einer Organspende kann allerdings nur die prospektive Erkennung potentieller Organspender wirklich beitragen. Wie im „Gemeinschaftlichen Initiativplan Organspende“ vorgeschlagen, könnten zukünftig evtl. Softwareprogramme (künstliche Intelligenz; Big-Data-Analysen) diese ebenso prospektiv unterstützen.

Für den Transplantationsbeauftragten sind verschiedene krankenhausspezifische Organisationsformen möglich. Wenn ein Entnahmekrankenhaus mehr als eine Intensivstation hat, soll, d.h. im Regelfall, für jede dieser Stationen mindestens ein Transplantationsbeauftragter bestellt werden ( $\$ \mathrm{gb}$ Abs. 1 TPG). Ist der Transplantationsbeauftragte zugleich z.B. der Oberarzt der Station, ist dieses einfach realisierbar. Im anderen Fall soll sichergestellt sein, dass die erste Erkennung potentieller Spender in allen intensivmedizinischen Bereichen durch qualifiziertes Personal eingeleitet und ein Transplantationsbeauftragter hinzugezogen wird. Insbesondere im Fall eines mehreren Stationen und Abteilungen übergeordneten Transplantationsbeauftragten in Voll- oder Teilzeit erscheint letzteres notwendig. Eine mehrmals tägliche aktive Visitation oder eine andere aktive systematische Suche des übergeordneten Transplantationsbeauftragten kann erwogen werden, letzteres wird teilweise in Empfehlungen anderer Länder als Expertenmeinung für sinnvoll erachtet (Westphal et al. 2016).

Entnahmekrankenhäuser bestellen mindestens einen qualifizierten ärztlichen Transplantationsbeauftragten ( $\$$ gb Abs. 1 TPG). Darüber hinaus sind auch qualifizierte pflegerische Transplantationsbeauftragte prinzipiell möglich, das Nähere hierzu wird allerdings durch das jeweilige Landesrecht (Landesausführungsgesetz) unterschiedlich geregelt.

\footnotetext{
Zur Realisierung einer Organspende, müssen als Schlüsselprozess potentielle Organspender prospektiv gemeinschaftlich vom Primärbehandlungsteam unter Hinzuziehung des Transplantationsbeauftragten erkannt werden.
} 


\title{
2.2 Verlaufsbeobachtung - klinische Symptome
}

\author{
Eckhard Rickels
}

Die engmaschige Beobachtung und neurologische Untersuchung von Patienten mit schwerer Hirnschädigung erfolgt, um wesentliche klinische Veränderungen auch während der Therapie frühzeitig erkennen zu können. Legen klinische oder apparative neurologische Befunde bzw. deren dynamische Entwicklung und Ausprägung einen progredienten Hirnfunktionsausfall nahe, so muss dies erkannt werden!

Um schwere Gehirnschäden zu behandeln und der Schädigung primär therapeutisch entgegenzuwirken, ist in der Regel eine Beatmung unter Analgosedierung notwendig. Sie erschwert aber die klinische Verlaufsbeobachtung, da unklar bleibt, ob die geminderte neurologische Antwort auf äußere Reize oder bei pflegerischen Maßnahmen den krankheitsbedingten IstZustand darstellt oder die Folge der medikamentösen Unterdrückung der Hirnfunktion (Sedierung) ist. Unter diesen Bedingungen ist das Erkennen eines schon eingetretenen oder sich anbahnenden irreversiblen Hirnfunktionsausfalls stark erschwert.

Es gibt jedoch einige Untersuchungsbefunde, die auch beim beatmeten und analgosedierten Patienten auf einen schon eingetretenen oder sich anbahnenden Hirnfunktionsausfall hinweisen.

Die Richtlinie der Bundesärztekammer zur Feststellung des endgültigen, nicht behebbaren Ausfalls der Gesamtfunktion des Großhirns, des Kleinhirns und des Hirnstamms gemäß $₫ 16$ Abs. 1, S. 1 N. 1 des TPG für Regeln zur Feststellung des Todes nach $\mathbb{3}$ der Bundesärztekammer betont die Gesamtfunktion des Gehirns und verlangt dabei die Feststellung des Gesamtausfalls aller prüfbaren Hirnfunktionen.

Auch die Untersuchung, um Anzeichen eines sich abzeichnenden oder vielleicht schon eingetretenen Hirnfunktionsausfalls zu erkennen, orientiert sich an dieser Richtlinie der Bundesärztekammer zur Feststellung des irreversiblen Hirnfunktionsausfalls. Während aber im Fall der „Hirntod-Diagnostik" der Beweis des Gesamtausfalls der Hirnfunktion erbracht werden muss, geht es beim Erkennen potenzieller Spender um das Erkennen einzelner Anzeichen, die auf den Funktionsausfall hinweisen. Nimmt der Summenwert bei Koma-Scores wie der Glasgow-Coma-Scale oder beim FOUR Score (Full-Outline of Unresponsiveness Score) ab, ist dies ebenfalls ein Indikator eines zunehmenden Hirnfunktionsausfalls. 
Auch ein sich kontinuierlich ausbreitender Funktionsausfall, eine langsame Abnahme der Antwort auf äußere Reize sind Ausdruck einer zunehmenden Verschlechterung der Hirnfunktion.

\section{Prinzipiell ist jede Verschlechterung der klinisch-neurologischen Untersuchungsbefunde, die nicht durch Analgosedierung erklär- bar ist, auf einen weiteren zusätzlichen Ausfall der Gehirnfunk- tionen verdächtig. Daraus ergeben sich Anzeichen, dass die Schädigung des Gehirns trotz Behandlung zunimmt.}

Die regelmäßige klinische Verlaufskontrolle konzentriert sich beim komatösen Patienten auf die neurologische Untersuchung auf Reflexantworten, die vom Hirnstamm und Diencephalon gesteuert werden. Schäden in diesen Arealen sind per se lebensbedrohlich. Fortschreitende Ausfälle müssen somit die Frage nach dem schon eingetretenen oder zu erwartenden irreversiblen Hirnfunktionsausfall aufwerfen.

Hinweise auf den eventuell schon eingetretenen, sich abzeichnenden oder bald zu erwartenden irreversiblen Hirnfunktionsausfall sind:

- Hirndruckanstieg: Am einfachsten zu erkennen ist der Funktionsausfall der Patienten, bei denen zurTherapiesteuerung eine Hirndrucksonde implantiert wurde - ein andauernder, nicht therapierbarer Hirndruckanstieg bei gleichzeitig weiten Pupillen wird den Verdacht auf eine zusätzliche schwere, lebensgefährdende Schädigung lenken. Ein CT wird ein Aufbrauchen aller Liquorreserveräume bei fehlender Abgrenzung von grauer und weißer Substanz zeigen.

- Cushing-Reflex: Ein plötzlicher extremer Blutdruckanstieg bei gleichzeitiger Bradykardie mit dem der Körper versucht, die Perfusion des Gehirns aufrecht zu erhalten wird als „Cushing-Reflex“ bezeichnet. Nach einiger Zeit erfolgt dann bei weiterhin weiten lichtstarren $\mathrm{Pu}$ pillen ein eindeutiger Blutdruckabfall.

- Diabetes insipidus: Eine durch die intensivmedizinische Therapie nicht erklärte Polyurie mit deutlicher Zunahme des Ausscheidungsvolumens und Abfall des spezifischen Uringewichts ist verdächtig auf eine Verminderung der Produktion des Antidiuretischen Hormons, ADH. Dies ist bedingt durch einen Ausfall von supraoptischen und paraventrikulären Strukturen des Hypothalamus.

- Hypothermie: Ein Absinken der Körpertemperatur, die nur durch Wärmezufuhr von außen stabilisiert werden kann, bedeutet eine Störung der körpereignen Temperaturregulation mit Ausfall der übergeordneten hypothalamischen Areale. 
- Hirnstammreflexe: Ausfall eines oder aller der Hirnnervenreflexe, die bei komatösen Patienten zu prüfen sind, aber deren Ausfall nicht medikamentös erklärt werden können:

n Weite lichtstarre Pupillen: Bei intaktem Regelkreis führt ein Lichteinfall ins Auge über die Verschaltung im Hirnstamm zu einer schnellen Engstellung der Pupillen. Eine Volumenzunahme des Gehirns durch Schwellung bedingt neben einer intrakraniellen Drucksteigerung, dass der Temporallappen den Nervus oculomotorius gegen den Tentoriumrand drückt. Der resultierende Funktionsausfall des Nervus oculomotorius mit Lähmung des parasympathisch innervierten Sphincter pupillae führt zum Überwiegen der aus dem Halssymphathicus kommenden Innervation des Dilatator pupillae. Unter den Bedingungen des schweren primären oder sekundären Hirnschadens sind beidseitig weite Pupillen als Ausdruck einer enormen, das ganze Hirn betreffenden, Hirnschwellung zu werten (Ausnahmen sind: Erblindung, Mydriaticum-Gabe, hochdosierte Gaben von Barbituraten mit dem Ziel der burst suppression im Rahmen der Hirndrucktherapie können zu weiten lichtstarren Pupillen führen!).

- Beidseitiges Fehlen des oculo-zephalen bzw. des vestibulo-oculären Reflexes: Eine abrupte Drehung des Kopfes des Patienten zur Seite führt zur Bewegung der Augen zur Gegenseite (Puppenkopfphänomen). Eine Spülung der Ohren mit kaltem Wasser führt zu ipsilateralen Augenbewegungen. Ein Ausbleiben dieser Antworten weist auf einen Schaden am Hirnstamm hin.

- Beidseitiges Fehlen des Cornealreflexes: Bei intaktem Regelkreis kommt es beim Berühren der Hornhaut mit einem Wattestäbchen zum Lidschluss beidseits.

- Fehlen von Reaktionen auf Schmerzreize: Beidseits im Trigeminusbereich und von zerebralen Reaktionen auf Schmerzreize außerhalb des Trigeminusbereichs - Schmerzen z. B. beim Lagern oder Schmerzen durch äußeren Reiz führen beim bewusstseinsgestörten Patienten zu sichtbaren Reaktionen. Das Gesicht wird verzogen, die Extremitäten werden bewegt, die Herzfrequenz steigt. Nehmen diese Reaktionen bei gleichbleibender Sedierungs- und Analgesietiefe ab, weist dies auf eine zusätzliche Hirnfunktionsstörung. Bei der Prüfung der Hirnfunktion wird die sensible Versorgung des Kopfes im Gesichtsbereich geprüft. Ein Schmerzreiz im Versorgungsgebiet des Nervus trigeminus (Der Versorgungsbereich des Trigeminus deckt den Gesichtsbereich bis zum Tragus ab) wird im Normalfall mit Grimassieren oder Herzfrequenzsteigerung beantwortet. Waren diese Reaktionen primär vorhanden und fallen im Verlauf aus, deutet das auf eine Verschlechterung hin. 
- Fehlen des Pharyngeal- und Trachealreflexes: Das Berühren der Rachenhinterwand und das Einführen eines Absaugkatheters in die Trachea führen zum Würgereiz und Husten. Ein neu auftretendes Ausbleiben dieser Reaktionen deutet auf eine Verschlechterung hin.

- Erlöschen einer Spastik, Auftreten einer Muskelhypotonie: Sofern keine Relaxierung vorliegt, zeigt sich die Tiefe eines Komas auch am Muskeltonus. Ein tiefes Koma (z.B. Mittelhirnsyndrom) zeigt neben der Pupillenstörung Beuge- und/oder Strecksynergismen. Im Übergang zum Hirnfunktionsausfall kommt es dann zum schlaffen Muskeltonus.

- Ausfall der Spontanatmung: Wenn anstelle der bislang unterstützenden Beatmung eine kontinuierliche maschinelle Beatmung notwendig wird, bedeutet dies eine Verschlechterung der Hirnstammfunktion mit Verdacht auf Schädigung des Atemzentrums. Eine Prüfung, ob ein endgültiger Ausfall der Spontanatmung vorliegt, sollte der Feststellung des irreversiblen Hirnfunktionsausfalls vorbehalten sein. Die Apnoe-Testung könnte zur Hirndrucksteigerung führen und sollte deshalb erst am Ende einer klinischen Prüfung des irreversiblen Hirnfunktionsausfalls durch die beiden Fachärzte erfolgen.

Parallel zu klinischen Veränderungen weisen Befunde aus der Bildgebung auf einen (drohenden) Hirnfunktionsausfall hin. Beim CCT sind es z.B. die Aufhebung der Rinden-Markgrenze, das vollständige Aufbrauchen der inneren und äußeren Liquorräume oder das Feststellen des Zirkulationsstillstandes im Rahmen einer CT-Angiographie. Neurophysiologische Untersuchungen wie z.B. evozierte Potenziale können auf eine neu aufgetretene Unterbrechung der Leitungsbahnen hinweisen, das EEG sich dem Nulllinien-EEG angleichen, der transkranielle Doppler-Ultraschall der hirnversorgenden Gefäße kann einen Pendelfluß zeigen.

Alle erwähnten technischen Befunde des Hirnfunktionsausfalls müssen nicht in ihrer Gesamtheit und nicht in voller Ausprägung auftreten.

Auch einzelne Veränderungen, die jeweils eine Verschlechterung der Hirnfunktion darstellen, können in der Gesamtbetrachtung von eigentlicher Erkrankung, Therapieoptionen und Krankheitsverlauf nahelegen, dass ein kuratives Therapiekonzept nicht mehr möglich ist und ein vollständiger Hirnfunktionsausfall sich etablieren wird. 
Laut Transplantationsgesetz (TPG $₫ 9 a$, Abs. 1) sind diese Patienten als potentieller Organspender zu betrachten. Nach $₫$ gb Abs. 1 Transplantationsgesetz kann und sollte bei potentiellen Organspendern der Transplantationsbeauftragte des Hauses hinzugezogen werden. Auch die Koordinierungsstelle der DSO kann bereits vor der eigentlichen Feststellung des irreversiblen Hirnfunktionsausfalls zur allgemeinen Beratung hinzugezogen werden ( $\$$ 9a, Abs. 2, Satz 1 TPG).

\title{
2.3 Therapieoptionen bei wahrscheinlichem Todeseintritt
}

\author{
Stefan Meier und Uwe Janssens
}

\subsubsection{Welche Therapieziele verbleiben bei wahrscheinlichem Todeseintritt?}

Unabhängig vom geäußerten Patientenwillen können sich Therapieziele nur an medizinisch indizierten Maßnahmen orientieren. Das bedeutet, dass in einigen medizinischen Situationen in Abhängigkeit von der bestehenden Prognose nur bestimmte Therapieziele denkbar sind. Liegt zum Beispiel eine Situation vor, bei der der Todeseintritt wahrscheinlich ist, ist zu klären, ob Therapieziele wie Heilung oder Lebensverlängerung nach professioneller Einschätzung überhaupt noch erreichbar sind (Neitzke et al. 2019). Wird diese Frage - idealerweise nach Diskussion aller an der Behandlung beteiligten ärztlichen und nichtärztlichen Behandler - mit „nein“ beantwortet, verbleibt als Therapieziel für den Fall, dass keine Organspende möglich ist, nur eine palliative Betreuung und (Sterbe-) Begleitung des Patienten und seiner Angehörigen. Liegt allerdings eine Situation vor, in der eine Organspende eine Option ist, muss mit den Angehörigen ein möglicher Organspendewunsch des Patienten frühzeitig geklärt werden (Bundesgesetzblatt 2020). Frühzeitig bedeutet in diesem Kontext, dass erste Gespräche schon vor der letztendlichen Feststellung des irreversiblen Hirnfunktionsausfalls (IHA) geführt werden sollen und nicht erst danach (Hahnenkamp et al. 2016).

Bei wahrscheinlichem Todeseintritt sind in aller Regel nur eine palliative Behandlung/Begleitung des Patienten oder-sofern die Vorrausetzungen dafür gegeben sind - die postmortale Organspende mögliche Therapieziele. 


\subsubsection{Therapieziel Organspende}

Die Besonderheit der Fortführung einer intensivmedizinischen Behandlung zum Zweck einer Organspende ist, dass diese Therapie nun nicht mehr primär dem Wohl des Patienten selbst dient, sondern dem der potenziellen Organempfänger. Um die Option der Organspende aufrecht zu erhalten ist es notwendig, die intensivmedizinische Therapie fortzuführen, während gleichzeitig mit den Angehörigen des Patienten der Patientenwille bezüglich einer potenziellen Organspende erörtert wird. Gespräche, in denen das mögliche Therapieziel Organspende thematisiert wird, bedürfen also sehr guter kommunikativer Fähigkeiten, um eine tragfähige und den Patientenwillen berücksichtigende Entscheidung zu ermöglichen.

Um besser zu verstehen, in welch komplexe Entscheidungssituationen gesetzliche Vertreter bzw. Angehörige (zur Unterscheidung s. Kap. 4, hier nur Angehörige) des Patienten kommen können, sei diese Situation im Folgenden noch einmal skizziert: Ist eine Organspende eine denkbare Option, werden die Angehörigen des Patienten, die in aller Regel noch unter dem schockierenden Eindruck der Nachricht vom wahrscheinlichen Versterben stehen, mit der Frage nach einer möglichen Organspende konfrontiert. Eine Frage, die (sofern nicht eine eindeutige Willensbekundung des Patienten wie zum Beispiel ein Organspendeausweis vorliegt) eine fast unmittelbare Entscheidung erfordert. Und zudem auch noch eine Frage, die es verlangt, jetzt nicht mehr nur auf den eigenen Angehörigen zu schauen, sondern noch andere Menschen wie die potenziellen Organempfänger mit in den Blick zu nehmen.

Aus dem Beschriebenen folgt, dass für diese Gespräche eine empathische und entlastende, die Entscheidungsfähigkeit stärkende Gesprächsführung notwendig ist (Jöbges et al. 2019). Es hat sich gezeigt, dass bei Gesprächen über das mögliche Therapieziel Organspende eine Thematisierung der wesentlichen Einflussfaktoren für eine Organspende hilfreich ist. Diese Einflussfaktoren lassen sich mit dem Akronym „DONATE“ zusammenfassen (Miller u. Breakwell 2018) (s. Tab. 1).

Demnach ist es hilfreich zum Beispiel gemeinsam mit den Angehörigen die Motivation des potentiellen Spenders in Bezug auf seinen Wunsch, anderen zu helfen, herauszuarbeiten. Dabei gilt es, den Sinn der möglichen Organspende aus Patientensicht zu verdeutlichen und damit in der gemeinsamen Entscheidungsfindung für die Angehörigen verstehbar machen, dass die Weiterführung der Therapie zur Organerhaltung und schließlich die Organspende auch zum Wohl des Verstorbenen sein kann. 
Tab. 1 DONATE-Konzept zur Strukturierung von Gesprächen zur Klärung der Option Organspende (nach Miller u. Breakwell 2018)

\begin{tabular}{|c|c|c|}
\hline Abkürzung & Bedeutung & Erläuterung \\
\hline \multicolumn{3}{|l|}{ DONATE } \\
\hline D & „Drivers" & Antrieb, der Familie zu helfen \\
\hline 0 & $\begin{array}{l}\text { „Optimal } \\
\text { communication“ }\end{array}$ & $\begin{array}{ll}\text { geschultes Personal } \\
\text { respektvoller Umgang } \\
\text { Gutes tun } \\
\text { Informieren }\end{array}$ \\
\hline N & $\begin{array}{l}\text { „Needs at time of } \\
\text { donation conversation" }\end{array}$ & n individuelle Bedürfnisse der Familie beachten \\
\hline A & „Altruism" & \\
\hline$T$ & „Timing“ & $\begin{array}{l}\text { Gesprächszeitpunkte individuell auf die Situation } \\
\text { des Spenders und der Familie anpassen }\end{array}$ \\
\hline E & „Empathy“ & $\begin{array}{l}\text { empathische Zuwendung und Unterstützung } \\
\text { positives Verstärken }\end{array}$ \\
\hline
\end{tabular}

Bei der Diskussion der Option „Organspende“ sollte sowohl den an der Behandlung beteiligten nichtärztlichen und ärztlichen Mitarbeitern als auch den Angehörigen des potentiellen Organspenders klar kommuniziert werden, dass es in der Phase bei wahrscheinlichem Todeseintritt darum geht, eine Stabilisierung der Organfunktionen bis zur definitiven Feststellung des IHA zu erreichen. Es muss deutlich werden, dass dieser Prozess und auch die Feststellung des IHA Zeit in Anspruch nimmt und die intensivmedizinische Behandlung dazu fortgeführt werden muss. Es sollte ebenso vermittelt werden, dass am Ende dieses Prozesses dann in der Regel die Organspende und erfolgreiche Transplantation der Spenderorgane steht, es aber bekanntermaßen aus verschiedensten Gründen auch denkbar ist, dass eine Spende nicht realisierbar ist.

Bei Gesprächen über die Therapieoption Organspende ist eine empathische und entlastende Gesprächsführung hilfreich, die die Entscheidungsfähigkeit der Patientenvertreter stärkt.

\subsubsection{Therapieziel Palliation}

Die erste und möglicherweise auch anspruchsvollste ärztliche Aufgabe bei Patienten mit wahrscheinlichem Todeseintritt besteht darin, den Angehörigen des Patienten auf empathische Art und Weise klarzumachen, dass es 
Tab. 2 Gesprächsstrategien zur Überbringung einer schlechten Nachricht: SPIKES (Baile et al. 2000) und VALUE (Lautrette et al. 2007; nach Jöbges et al. 2019). IHA = Irreversibler Hirnfunktionsausfall

\begin{tabular}{|c|c|c|}
\hline Abkürzung & Kommunikation & Erläuterung \\
\hline \multicolumn{3}{|l|}{ SPIKES } \\
\hline S & „Setup“ & $\begin{array}{l}\text { angenehme Gesprächsatmosphäre } \\
\text { alle wesentlichen Personen sollten } \\
\text { anwesend sein } \\
\text { inhaltlich gut vorbereitet }\end{array}$ \\
\hline$P$ & „Perception“ & $\begin{array}{l}\text { Verständnis/Wissensstand der aktuellen } \\
\text { Situation erfragen }\end{array}$ \\
\hline I & „Invitation“ & $\begin{array}{l}\text { Ermitteln des Informationsbedürfnisses } \\
\text { der Familie }\end{array}$ \\
\hline K & „Knowledge“ & $\begin{array}{l}\text { Informationsvermittlung: } \\
\text { bisherige Behandlungsversuche und warum } \\
\text { diese nicht wirkten } \\
\text { Bedeutung des IHA und Prognose }\end{array}$ \\
\hline E & „Empathize“ & - den Emotionen der Familie Zeit und Raum geben \\
\hline$S$ & $\begin{array}{l}\text { "Summarize and } \\
\text { strategize" }\end{array}$ & $\begin{array}{l}\text { beenden des Gesprächs durch Zusammenfassung } \\
\text { des Besprochenen }\end{array}$ \\
\hline \multicolumn{3}{|l|}{ VALUE } \\
\hline V & „Value“ & wertschätzende und respektvolle Kommunikation \\
\hline A & „Acknowledge“ & $\begin{array}{l}\text { Anerkennen der Gefühle und Einstellungen } \\
\text { der Angehörigen }\end{array}$ \\
\hline L & „Listen“ & - Zuhören, Angehörige (aus-)reden lassen \\
\hline U & „Understand“ & $\begin{array}{l}\text { den Patienten als Menschen verstehen und } \\
\text { darüber reden (lassen) }\end{array}$ \\
\hline E & „Elicit“ & zu Fragen ermuntern \\
\hline
\end{tabular}

zu einem Versterben des Patienten kommen wird. Es gilt zu verdeutlichen, dass es - sofern eine Organspende nicht in Frage kommt - außer einer palliativen Begleitung und symptomkontrollierenden Behandlung kein weiteres Therapieziel geben wird. Gute kommunikative Fähigkeiten, die auch bewährte Kommunikationshilfen wie das SPIKES-Modell (Baile et al. 200o) oder die Value-Kriterien (Lautrette et al. 2007) zum Überbringen schwieriger Nachrichten nutzen, sind notwendig, um in diesen Situationen angemessen zu handeln (s. Tab. 2). 
Gerade in komplexeren Behandlungssituationen ist eine frühzeitige palliativmedizinische Mitbehandlung des Patienten durch palliativmedizinisch ausgebildete ärztliche und nichtärztliche Mitarbeiter sinnvoll (Adler et al. 2017). Denkbare Themenkomplexe dieser im Idealfall multiprofessionellen Mitbehandlung sind dann unter anderem die Unterstützung in schwierigen Gesprächssituationen, Hilfe bei der symptomkontrollierenden Therapie und die psychologische Unterstützung der Angehörigen, die auch über den Zeitpunkt des Versterbens des Patienten fortgeführt werden kann. Über die in diesem Beitrag thematisierte Situation des wahrscheinlichen Todeseintritts hinaus hat sich herausgestellt, dass es vorteilhaft sein kann, die palliative Mitbehandlung schon frühzeitig, so zum Beispiel in Situationen, in denen die Prognose unsicher und ein Versterben denkbar, aber eben noch nicht unbedingt schon wahrscheinlich ist, zu beginnen (Meier $\mathrm{u}$. Nauck 2020).

Wenn verfügbar, kann eine Einbindung palliativmedizinischer Expertise bei der Versorgung von Patienten mit wahrscheinlichem Todeseintritt hilfreich sein.

\section{Literatur}

Adler K, Schlieper D, Kindgen-Milles D et al. (2017) Integration der Palliativmedizin in die Intensivmedizin: Systematische Übersichtsarbeit. Anaesthesist 66:660-666

Baile WF, Buckman R, Lenzi R et al. (2000) SPIKES-A six-step protocol for delivering bad news: application to the patient with cancer. Oncologist 5:302-311

Bender A et al. (2018) S1-Leitlinie Hypoxisch-ischämische Enzephalopathie im Erwachsenenalter. In: Deutsche Gesellschaft für Neurologie (Hrsg.) Leitlinien für Diagnostik und Therapie in der Neurologie. URL: https://www.awmf.org/uploads/tx_szleitlinien/030-119_S1_Hypoxisch-ischaemischeEnzephalopathie-HIE_2018-03.pdf (abgerufen am 02.07.2021)

Bundesärztekammer, Zentrale Ethikkommission bei der Bundesärztekammer (2013) Arbeitspapier zum Verhältnis von Patientenverfügung und Organspendeerklärung. Deutsches Ärzteblatt, 110, 2013(12), A572-A574. URL: https://www.aerzteblatt.de/pdf.asp?id=135909 (abgerufen am 02.07.2021)

Bundesgesetzblatt (2020) Gesetz zur Stärkung der Entscheidungsbereitschaft bei der Organspende vom 16. März 2020. Bundesgesetzblatt Jahrgang 2020 Teil I Nr. 13, ausgegeben zu Bonn am 19.03. 2020. URL: http://www.bgbl.de/xaver/bgbl/start.xav?startbk=Bundesanzeiger_BGBI\&jumpTo=bgbl120s0497.pdf (abgerufen am 07.07.2021)

Childress JF, Beauchamp TL (2008) Principles of biomedical ethics. 6. Aufl. OxfordUniversityPress, NewYork

Dominguez-Gil B, Delmonico F, Shaheen FAM et al. (2011) The critical pathway for deceased donation: reportable uniformity in the approach to deceased donation. Transpl Int. 24(4): p. 373-8

Escudero D, Otero I, de León BM et al. (2017) Organ Donation and Elective Ventilation: A Necessary Strategy. Biomed Res Int. 2017: p. 7518375 
Gardiner D, Shaw DM, Kilcullen JK et al. (2019) Intensive care for organ preservation: A four-stage pathway. I Intensive Care Soc. 20(4): p. 335-340

Guide to the Quality and Safety of Organs for Transplantation (2018) Strasbourg: European Directorate for the Quality of Medicines \& HealthCare (EDQM), Council of Europe. 7. Auflage (Kapitel 2). URL: https://www.edqm.eu/en/news/new-release-7th-edition-guide-quality-and-safety-organs-transplantation (abgerufen am 02.07.2021)

Hahnenkamp K, Janssens U, Beckmann M et al. (2016) Entscheidung bei potentiellen Organspendern. Gemeinsames Positionspapier der Sektion Ethik und der Sektion Organspende und -transplantation der DIVI. Anästh Intensivmed. 57:152-154

Harvey D, Butler I, Groves / et al. (2018) Management of perceived devastating brain injury after hospital admission: a consensus statement from stakeholder professional organizations. $\mathrm{Br}$ | Anaesth. 120(1): p. 138-145

Janssens U (2020) Ethicus-2: Therapie am Lebensende auf europäischen Intensivstationen im Wandel der Zeit. Intensiv-News (3) 2020: 28-30

Jöbges S, Denke C, Kumpf 0 et al. (2019) Gesprächsführung mit Angehörigen. Med Klin Intensivmed Notfmed 114:122-127

Lautrette A, Darmon M, Megarbane B et al. (2007) A communication strategy and brochure for relatives of patients dying in the ICU. N.Engl.J.Med. 356:469-478

Martin-Loeches I, Sandiumenge A, Charpentier / et al. (2019) Management of donation after brain death (DBD) in the ICU: the potential donor is identified, what's next? Intensive Care Med. 45(3): p. $322-330$

Medizin-ethische Richtlinien der Schweizerischen Akademie für Medizinische Wissenschaften (SAMW) zur „Feststellung des Todes im Hinblick auf Organtransplantationen und Vorbereitungen der Organentnahme" vom 16. Mai 2017. Praktische Umsetzung (Kapitel 2.3.1). URL: https://www. samw.ch/dam/jcr:436e4826-d071-42b6-a26e-a0b81321e3do/ (abgerufen am 02.07.2021)

Meier S, Nauck F (2020) Aspekte der Palliativmedizin für die Intensivmedizin. DIVI 11:116-123

Miller C, Breakwell R (2018) What factors influence a family's decision to agree to organ donation? A critical literature review. London I Prim Care (Abingdon) 10:103-107

Neitzke G, Burchardi H, Duttge G et al. (2019) Grenzen der Sinnhaftigkeit von Intensivmedizin: Positionspapier der Sektion Ethik der DIVI. Med Klin Intensivmed Notfmed 114:46-52

Neitzke G, Rogge A, Lücking KM et al. (2019) Decision-making support in Intensive Care to facilitate organ donation: Position paper of the Ethics Section and the Organ Donation and Transplantation Section of the German Interdisciplinary Association of Critical Care and Emergency Medicine (DIVI) in collaboration with the Ethics Section of the German Society of Medical Intensive Care Medicine and Emergency Medicine (DGIIN). Med Klin Intensivmed Notfmed. 114(4): p. 319-326

OPTN Organ Procurement and Transplantation Network (2017) Policies. URL: http://optn.transplant. hrsa.gov/media/1200/optn_policies.pdf (abgerufen am 02.07.2021)

Rohaut B, Claassen I (2018) Decision making in perceived devastating brain injury: a call to explore the impact of cognitive biases. Br | Anaesth. 120(1): p. 5-9

Schulte K, Borzikowsky C, Rahmel A et al. (2018) Decline in Organ Donation in Germany. Dtsch Arztebl Int. 115(27-28): p. 463-468

Souter MJ, Blissitt PA, Blosser S et al. (2015) Recommendations for the Critical Care Management of Devastating Brain Injury: Prognostication, Psychosocial, and Ethical Management: A Position Statement for Healthcare Professionals from the Neurocritical Care Society. Neurocrit Care. 23(1): p. 4-13

Sprung CL, Ricou B, Hartog CS (2019) Changes in End-of-Life Practices in European Intensive Care Units From 1999 to 2016. Jama. 322(17): p. 1-12 
Squires JE, Coughlin M, Dorrance K et al. (2018) Criteria to Identify a Potential Deceased Organ Donor: A Systematic Review. Crit Care Med. 46(8): p. 1318-1327

Westphal GA, Garcia VD, de Souza RL et al. (2016) Guidelines for the assessment and acceptance of potential brain-dead organ donors. Rev Bras Ter Intensiva. 28(3): p. 220-255

Zavalkoff S, Shemie SD, Grimshaw IM et al. (2019) Potential organ donor identification and system accountability: expert guidance from a Canadian consensus conference. Can I Anaesth. 66(4): p. $432-447$ 